\title{
Sphingosine-1-Phosphate Is a Key Regulator of Proliferation and Differentiation in Retina Photoreceptors
}

\author{
Gisela E. Miranda, Carolina E. Abraban, Luis E. Politi, and Nora P. Rotstein
}

Purpose. Identifying the cues required for the survival and development of photoreceptors is essential for treating retinal neurodegeneration. The authors previously established that glial-derived neurotrophic factor (GDNF) stimulates proliferation and that docosahexaenoic acid (DHA) promotes photoreceptor survival and differentiation. Later findings that ceramide triggers photoreceptor apoptosis suggested sphingolipids might also control photoreceptor development. The present study investigated whether sphingosine-1-phophate (S1P), which promotes survival and differentiation in several cell types, regulates photoreceptor proliferation and differentiation and whether it is a mediator in GDNF and DHA effects.

Methods. Rat retina neuronal cultures were supplemented at day 0 or 1 with S1P, GDNF, or DHA and were treated with DL-threo-dihydrosphingosine to inhibit S1P synthesis or with brefeldin A (BFA) to block intracellular trafficking. Proliferation was quantified to determine bromodeoxyuridine uptake and number of mitotic figures. Opsin, peripherin, and sphingosine kinase (SphK), the enzyme required for S1P synthesis, were quantified by immunocytochemistry and Western blot analysis.

RESults. S1P increased the proliferation of photoreceptor progenitors. It also stimulated the formation of apical processes, enhanced opsin and peripherin expression, and promoted their localization in these processes; DHA had similar effects. BFA prevented S1P and DHA enhancement of apical process formation without affecting opsin expression. GDNF and DHA enhanced SphK expression in photoreceptors, while inhibiting S1P synthesis blocked GDNF mitogenic effects and DHA effects on differentiation.

Conclusions. The authors propose $\mathrm{S} 1 \mathrm{P}$ as a key regulator in photoreceptor development. GDNF and DHA might upregulate SphK levels to promote S1P synthesis, which would initially promote proliferation and then advance photoreceptor differentiation. (Invest Ophthalmol Vis Sci. 2009;50:4416-4428) DOI:10.1167/iovs.09-3388

From the Instituto de Investigaciones Bioquímicas de Bahía Blanca, Universidad Nacional del Sur-CONICET, Bahía Blanca, Buenos Aires, Argentina.

Supported by grants from FONCyT; the Argentine National Research Council (CONICET); and the Universidad Nacional del Sur, Bahia Blanca, Argentina.

Submitted for publication January 8, 2009; revised February 24 and March 12, 2009; accepted June 9, 2009.

Disclosure: G.E. Miranda, None; C.E. Abrahan, None; L.E. Politi, None; N.P. Rotstein, None

The publication costs of this article were defrayed in part by page charge payment. This article must therefore be marked "advertisement" in accordance with 18 U.S.C. $\$ 1734$ solely to indicate this fact.

Corresponding author: Nora P. Rotstein, Instituto de Investigaciones Bioquímicas de Bahía Blanca, Universidad Nacional del SurCONICET, CC 857, B8000FWB Bahía Blanca, Argentina;

inrotste@criba.edu.ar.
$\mathbf{R}$ od and cone photoreceptors of the vertebrate retina are complex neurons designed for the task of receiving light. For this purpose photoreceptors develop specialized structures-the outer segments (OS) - highly enriched in the visual pigment opsin and in docosahexaenoic acid (DHA), a polyunsaturated fatty acid. Development and preservation of an adequate number of photoreceptors and, more particularly, of OS are crucial not only for proper vision but also for photoreceptor survival. Genetic mutations deriving from improper development and faulty assembly of OS significantly contribute to the different phenotypes leading to photoreceptor apoptosis, a common feature of many neurodegenerative diseases of the retina. No effective treatments are available for inherited neurodegenerative diseases of the retina. Several trophic factors, such as fibroblast growth factor, ciliary neurotrophic factor, and glial-derived neurotrophic factor (GDNF), ${ }^{1,2}$ partially prevent the apoptosis of photoreceptors and advance their differentiation. We have previously demonstrated that GDNF and DHA promote photoreceptor survival and differentiation in vitro. ${ }^{3-6}$ They also play roles in controlling the proliferation of photoreceptor progenitors; GDNF enhances this proliferation, whereas DHA promotes the exit of neuroblasts from the cell cycle and stimulates their differentiation as photoreceptors. ${ }^{7}$ Molecular clues and intracellular pathways involved in these effects have still to be uncovered.

Possible mediators in these pathways might be a new family of signaling molecules, the sphingolipids; attention has been focused in recent years on molecules such as ceramide, sphingosine, and sphingosine-1-phosphate (S1P), which have been shown to control cell death, survival, proliferation, and development in many cell systems. ${ }^{8-13}$ Knowledge on the roles of these lipids in the retina, particularly in photoreceptors, is scarce. Recent work has demonstrated that ceramide is a key mediator in the induction of photoreceptor death. ${ }^{14,15}$ Oxidative stress induces an increase in ceramide levels that leads to photoreceptor apoptosis; conversely, blocking ceramide synthesis prevents this death. ${ }^{14}$ Moreover, DHA protects photoreceptors from ceramide-induced apoptosis and inhibition of glucosylceramide synthase, which catalyzes ceramide glucosylation, thus decreasing ceramide levels, blocks DHA protection. ${ }^{14}$ This suggested that other sphingolipids might also play crucial functions in directing several aspects of photoreceptor development.

$\mathrm{S} 1 \mathrm{P}$ is a bioactive lipid that regulates a wide range of biological functions, including cell growth and survival, proliferation, neuritogenesis, cell migration, angiogenesis, and immune function. ${ }^{16,17}$ Its cellular levels are regulated by the enzymes involved in its synthesis and degradation. S1P is synthesized by the phosphorylation of sphingosine catalyzed by sphingosine kinase (SphK), which has two mammalian isoforms, SphK1 and SphK2. Exogenous expression of SphK1 leads to cell proliferation and prosurvival signals, whereas its downregulation with siRNA induces proapoptotic events. ${ }^{18}$ Different trophic factors enhance S1P levels by stimulating SphK expression or activation ${ }^{19,20}$ to promote cell survival. 
Little is known concerning S1P roles in the nervous system; it is involved in brain neurogenesis ${ }^{21}$ and promotes the proliferation of neuronal progenitors in embryonic rat brain. ${ }^{22}$ Information on S1P functions in the retina is even scarcer. It has been proposed to participate in rhodopsin trafficking to the OS, ${ }^{23}$ and lipid phosphatases involved in its hydrolysis have been described in rod OS. ${ }^{24}$

In this study, we investigated whether S1P participated in the regulation of photoreceptor development in culture. Our results show for the first time that S1P enhanced the proliferation of photoreceptor progenitors and promoted their differentiation. These results also suggest that S1P might act as a second messenger for trophic factors, such as DHA and GDNF, which would enhance its synthesis to regulate the final number of photoreceptors and control their development.

\section{Materials ANd Methods}

\section{Materials}

Albino Wistar rats bred in our own colony were used in all the experiments. All proceedings concerning animal use were performed in accordance with the ARVO Statement for the Use of Animals in Ophthalmic and Vision Research. Plastic culture 35- and 60-mm diameter dishes (CellStar) were from Greiner Bio-One (Frickenhausen, Germany). Dulbecco modified Eagle medium (DMEM), trypsin, insulin, gentamicin, 5-bromo-2-deoxyuridine (BrdU), 5-bromo-2'-deoxyuridine5 '-triphosphate (BrdUTP), and terminal deoxynucleotidyl transferase (TdT) were from Invitrogen (Carlsbad, CA). Bovine serum albumin (Fraction V; fatty acid-free; low endotoxin, tissue culture tested), polyL-ornithine, trypsin inhibitor, transferrin, hydrocortisone, putrescine, 4,6-diamidino-2-phenylindole (DAPI), tyramine, docosahexaenoic acid (DHA), paraformaldehyde, and monoclonal anti-acetylated $\alpha$-tubulin antibody were from Sigma Chemical Co. (St. Louis, MO). S1P was from Calbiochem (Merck Chemicals, Darmstadt, Germany). Brefeldin A, kainic acid, DL-threo-dihydrosphingosine (DHS) and MG-132 were from Biomol (Plymouth Meeting, PA). Monoclonal antibody against BrdU (clone G3G4) was from DSHB (developed under the auspices of the NICHD and maintained by The University of Iowa, Department of Biological Sciences, Iowa City, IA). Secondary antibody, Cy2-conjugated goat anti-rabbit, was from Jackson ImmunoResearch (West Grove, PA). Monoclonal antibody anti-actin and secondary antibodies used for Western blot analysis, goat anti-mouse IgG-horseradish peroxidase (HRP), and goat anti-rabbit IgG-HRP, were from Santa Cruz Biotechnology Inc. (Santa Cruz, CA). Fluorophore-conjugated tyramine compounds and reaction buffers were synthesized according to previous reports. ${ }^{25,26}$ Secondary antibodies biotin-conjugated goat antirabbit and avidin-conjugated horseradish peroxidase were from Vector Laboratories (Burlingame, CA). GDNF was from PeproTech (Rocky Hill, NJ) and a kind gift from Néstor Carri (IMBICE, Buenos Aires, Argentina). Monoclonal antibodies anti-opsin (Rho4D2) and anti-peripherin (clone Per3B6) were generous gifts from Robert Molday (University of British Columbia, Canada). Polyclonal antibody anti-SphK1 was kindly provided by Lina Obeid (Medical University of South Carolina). Solvents were HPLC grade, and all other reagents were analytical grade.

\section{Neuronal Cultures}

Purified cultures of rat retinal neurons were prepared as previously described. ${ }^{5,27}$ Approximately $0.5 \times 10^{5}$ cells $/ \mathrm{cm}^{2}$ were seeded on 35 -mm diameter dishes, pretreated with polyornithine and Schwannomaconditioned medium, ${ }^{28}$ and cultured in a chemically defined medium. ${ }^{27}$ Proliferation was analyzed in neuronal cultures prepared from day 0 rat retinas, in which neuroblast proliferation is active ${ }^{7}$; differentiation was evaluated in cultures obtained from 2-day-old rat retinas.

\section{Addition of S1P and DHA}

Aliquots from a S1P stock solution $(0.5 \mathrm{mg} / \mathrm{mL}$, in methanol/water, 95:5) were evaporated under a stream of dry nitrogen, resuspended in a bovine serum albumin (BSA) solution in DMEM $(4 \mathrm{mg} / \mathrm{mL})$, and heated at $40^{\circ} \mathrm{C}$ to $50^{\circ} \mathrm{C}$ for 30 minutes with occasional vortexing and sonication to allow solubilization. S1P effect on opsin expression was evaluated at concentrations ranging from 0.5 to $5 \mu \mathrm{M}$. A final $1 \mu \mathrm{M} \mathrm{S} 1 \mathrm{P}$ concentration (in the culture medium) was chosen for subsequent experiments. The same volume of the solution used as vehicle was added to controls.

DHA $(6.7 \mu \mathrm{M})$ was added to day 1 cultures, complexed with BSA, in a 2:1 (DHA/ BSA) molar ratio. ${ }^{5}$ The same volume of the BSA solution was added to controls.

\section{Evaluation of Neuroblast Proliferation}

To investigate the S1P effect on the proliferation of photoreceptor progenitors, neuronal cultures were supplemented with S1P 1 hour after cells were seeded, and proliferation was evaluated 1 or 2 days later. BrdU uptake was determined by incubating 0- and 1-day cultures with $30 \mu \mathrm{M}$ BrdU (final concentration in culture) for 16 to 18 hours. Cells were fixed for at least 30 minutes, treated with $2 \mathrm{~N} \mathrm{HCl}$ for 30 minutes for DNA denaturation, and neutralized with $0.1 \mathrm{M}$ boric acid. BrdU uptake was determined using a monoclonal antibody against BrdU. Mitotic figures were evaluated by fluorescence microscopy after cells were permeated with $0.1 \%$ Triton X-100 in PBS and incubated for 20 minutes with DAPI, a DNA marker.

\section{Addition of Brefeldin A}

A stock solution of the fungal antibiotic brefeldin A (BFA; $4 \mathrm{mg} / \mathrm{mL}$ ) was prepared in dimethylsulfoxide; dilutions (using Hanks balanced salt solution) were then prepared. Day 2 neuronal cultures were treated with $0.5 \mu \mathrm{g} / \mathrm{mL}$ BFA (final concentration); day 5 cultures were treated with $0.25 \mu \mathrm{g} / \mathrm{mL}$ BFA (final concentration) to avoid deleterious effects; DHA or S1P, or both were added 2 hours later, and cells were fixed at day 4 or 6 , respectively. Photoreceptor differentiation was then evaluated.

\section{Immunocytochemical Methods}

After different time periods, cultures were fixed for at least 1 hour with $2 \%$ paraformaldehyde in PBS at room temperature, followed by permeation with Triton X-100 (0.1\% in PBS) for 15 minutes. Photoreceptors were identified by immunocytochemistry with the monoclonal antibody Rho4D2, by their morphology, and by other criteria as previously described. ${ }^{6,29}$ Cy2-conjugated goat anti-mouse was used as the secondary antibody. Amplification with tyramine fluorescent compounds was occasionally used to improve visualization. Controls for immunocytochemistry were made by omitting the primary or the secondary antibody.

Cultures were then analyzed by phase and fluorescence microscopy (Eclipse E600 microscope; Nikon, Tokyo, Japan) with a condenser (C-C Phase-Contrast Turret; Technical Instruments, Burlingame, CA) and an epifluorescence attachment (Y-FL; Nikon) and by laser scanning confocal microscopy (DMIRE2/TSCSP2 microscope; Leica, Wetzlar, Germany) with a $63 \times$ water objective; $x-y$ (top to bottom) sections were collected and processed (LCS software; Leica).

\section{Evaluation of Photoreceptor Differentiation}

Opsin and peripherin expression and the amount of photoreceptors with either opsin or peripherin positive-apical processes were evaluated using Rho4D2 and anti-peripherin monoclonal antibodies, respectively. ${ }^{30,31}$ Cy2-conjugated goat anti-mouse secondary antibody and amplification with tyramine fluorescent compounds were used for opsin and peripherin detection, respectively. For double immunostaining for opsin and peripherin and for acetylated $\alpha$-tubulin and periph- 
erin, a modification of the protocol described by Uchihara et al. ${ }^{32}$ was used.

\section{Selective Elimination of Amacrine Neurons}

To evaluate different parameters exclusively in photoreceptors, amacrine neurons were eliminated from the cultures by previously established methods. ${ }^{33}$ In brief, 3- or 4-day cultures were incubated overnight with $0.25 \mathrm{mM}$ kainic acid, which selectively kills amacrine neurons while photoreceptors remain unaffected. Cells were then fixed or lysed.

\section{Inhibition of S1P Synthesis}

To investigate whether GDNF promoted S1P synthesis to stimulate proliferation, day 0 cultures were supplemented with $1 \mu \mathrm{M}$ DHS, a potent, cell-permeable, competitive inhibitor of SphK1, ${ }^{4,35}$ and 30 minutes later without or with GDNF ( $4 \mathrm{ng} / \mathrm{mL}$, final medium concentration). BrdU was added 4 hours later, and cells were fixed after 18-hour incubation. Proliferation was evaluated as described.

To evaluate whether the effect of DHA on photoreceptor differentiation required S1P synthesis, 1 day-cultures were treated with DHS and were supplemented 1 hour later with or without DHA or S1P. Photoreceptor differentiation was evaluated at day 6 .

\section{Effect of DHA and GDNF on SphK Levels}

To analyze the effect of GDNF on SphK expression, GDNF was added 1 hour after the cells were seeded. At this time in development, kainic acid is not toxic for amacrine neurons; hence, SphK expression in amacrine and photoreceptor cells was determined. To evaluate the effect of DHA on SphK expression in photoreceptors, amacrine neurons were eliminated, as described, and fresh media with or without DHA were added at day 4 . Neurons were fixed or lysed 24 hours after the addition of DHA or GDNF. To improve the detection of SphK, its proteosomal degradation was inhibited by the treatment of cultures with the proteosome inhibitor MG-132 $(0.1 \mu \mathrm{M})^{36} 18$ hours before fixation or lysis. SphK expression was evaluated by immunocytochemistry or Western blot analysis with the use of a specific polyclonal antibody, anti-SphK1.

\section{Protein Extraction and Western Blot Analysis}

Expression of opsin, peripherin, and SphK was evaluated by Western blot analysis. Media were removed. Cells were rinsed with PBS, collected in lysis buffer $(3 \mathrm{mM} \mathrm{KCl}, 50 \mathrm{mM}$ Tris-HCl, $\mathrm{pH}$ 7.4, $150 \mathrm{mM}$ NaCl, $1 \mathrm{mM}$ EDTA, 1\% Tween-20, 1\% NP-40) containing a protease inhibitor mixture, and lysed in ice for 20 minutes. Proteins were quantified with a protein assay (DC; Bio-Rad; Hercules, CA) based on the Lowry assay and were separated by one-dimensional SDS-PAGE. ${ }^{37}$ Briefly, samples were mixed with $6 \times$ Laemmli sample buffer and were heated for 5 minutes at $95^{\circ} \mathrm{C}$ for $\mathrm{SphK}$ or not heated for opsin and peripherin. Proteins (either 10 or $20 \mu \mathrm{g} / \mathrm{sample}$ ) were subjected to electrophoresis on $10 \%$ SDS-polyacrylamide minigels and then transferred to polyvinylidene difluoride (Immobilon P; Millipore, Billerica, MA) membranes. Membranes were then washed in buffer with 5\% nonfat dry milk for 1 hour at room temperature to block nonspecific binding. Anti-opsin, anti-peripherin, anti-SphK1, and anti-actin antibodies were allowed to react with the membrane overnight at $4{ }^{\circ} \mathrm{C}$ or for 2 hours at room temperature. Membranes were thoroughly washed and incubated with horseradish peroxidase-conjugated goat antimouse or anti-rabbit secondary antibodies, in TBS-T for 1 hour at room temperature and were then visualized with enhanced chemiluminescence. Images (not shown) were obtained by scanning at $600 \mathrm{dpi}$. Bands were quantified with ImageJ software (developed by Wayne Rasband, National Institutes of Health, Bethesda, MD; available at http://rsb.info.nih.gov/ij/index.html).

\section{Evaluation of Photoreceptor Apoptosis}

Apoptosis was determined at day 6 by terminal deoxynucleotide transferase dUTP nick end labeling (TUNEL) staining and by evaluating nuclei integrity. For TUNEL staining, the cultures were fixed with $2 \%$ paraformaldehyde and stored in $70 \%$ ethanol for 48 hours at $-20^{\circ} \mathrm{C}$. Cells were then preincubated with $1 \times \mathrm{TdT}$ buffer for 15 minutes and with the TdT reaction mixture $(0.05 \mathrm{mM}$ BrdUTP and $0.3 \mathrm{U} / \mu \mathrm{L}$ TdT in TdT buffer) at $7^{\circ} \mathrm{C}$ in a humidified atmosphere for 1 hour. The reaction was stopped with stop buffer $(300 \mathrm{mM} \mathrm{NaCl}, 30 \mathrm{mM}$ sodium citrate, $\mathrm{pH}$ 7.4) at room temperature. Negative controls were prepared by omitting TdT. The presence of BrdU was determined with an antiBrdU monoclonal antibody.

Nuclei integrity was evaluated after staining cell nuclei with DAPI, as described. Cells were considered apoptotic when they showed fragmented or condensed (pyknotic) nuclei. The percentage of apoptotic photoreceptors was determined by double-labeling with DAPI and Rho4D2 to unambiguously identify cells as photoreceptors and to establish the total number of these cells.

\section{Statistical Analysis}

For cytochemical studies, 10 fields per sample, randomly chosen, were analyzed in each case. Each value represents the average of at least three experiments, with three or four dishes for each condition \pm SD. Statistical significance was determined by Student's two-tailed $t$-test.

\section{Results}

\section{Effect of S1P on the Proliferation of Photoreceptor Precursors}

In our culture conditions, photoreceptor progenitors proliferated for 1 to 2 days before differentiating as photoreceptors ${ }^{7}$; therefore, we investigated whether S1P promoted this proliferation. S1P addition at day 0 rapidly increased BrdU uptake in day 1 progenitors (Figs. 1A-D), from $23 \%$ in controls to $33 \%$ in S1P-supplemented cultures (Fig. 1E). S1P also augmented the amount of mitotic figures, which were more than 2.5-fold higher in S1P-supplemented cultures than in controls (Fig. 1F). In normal conditions, proliferation decreased rapidly; in 2-day controls, few neuroblasts took up BrdU, and almost no mitotic cells were present. BrdU-labeled cells (Fig. 1E) and mitotic figures (Fig. 1F) were still present in S1P-supplemented cultures, suggesting S1P preserved some mitotic activity.

\section{Effect of S1P on Photoreceptor Differentiation}

Retinal photoreceptors in vitro, cultured in media lacking their trophic factors, develop as round cells with small bodies and short cilia, ${ }^{5,29}$ but their development seems to be hindered compared with their in vivo counterparts. They usually lack the high opsin levels and characteristic OS found in photoreceptors in vivo; few cultured photoreceptors express opsin or develop apical processes that resemble rudimentary OS. DHA supplementation enhances opsin expression and the development of apical processes and promotes opsin localization in these processes. ${ }^{3,5,38}$ Adding S1P at day 1 rapidly enhanced opsin expression; a slight but significantly higher percentage of photoreceptors expressing opsin, compared with controls, was already observed 4 hours after this addition (value corresponding to day 1 in Fig. 2A) and increased over time. S1P and DHA had similar effects on opsin levels, which followed the same time course of increase. Their combined addition at day 1 increased opsin expression compared with controls but had no additive effect. By day 6, cultures with S1P and DHA 
Ctl

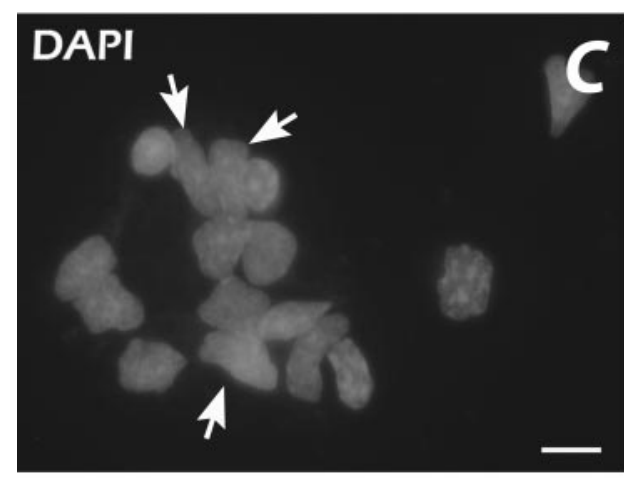

Figure 1. Effect of S1P on the proliferation of photoreceptor progenitors. Neuronal cultures, prepared from retinas obtained from P0 rats, were supplemented with $1 \mu \mathrm{M} \mathrm{S1P}$ or with the bovine serum albumin (BSA) solution (Ctl) in which S1P was resuspended 1 hour after seeding of the cells. BrdU was added to the cultures 4 or 30 hours later, and its uptake was determined after 18 hours using a specific monoclonal antibody. Fluorescence micrographs show BrdU uptake (A, B) and nuclei labeled with DAPI $(\mathbf{C}, \mathbf{D})$ in 1-day control (left) and S1P-supplemented $(r i g h t)$ cultures. Note the mitotic figures (B, D, arrowheads). Percentages of BrdU-positive cells (E) in 1and 2-day cultures, with or without S1P. Amount of mitotic figures (F) observed in photoreceptor progenitors after 1 and 2 days in culture, with or without S1P. ${ }^{*} P<0.05$; statistically significant differences compared with controls.

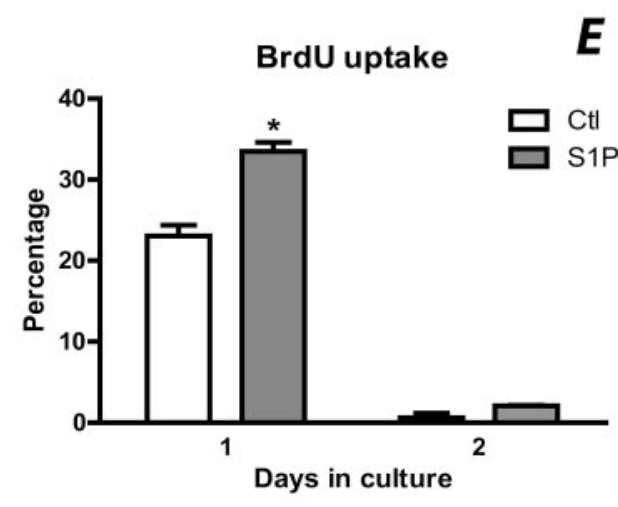

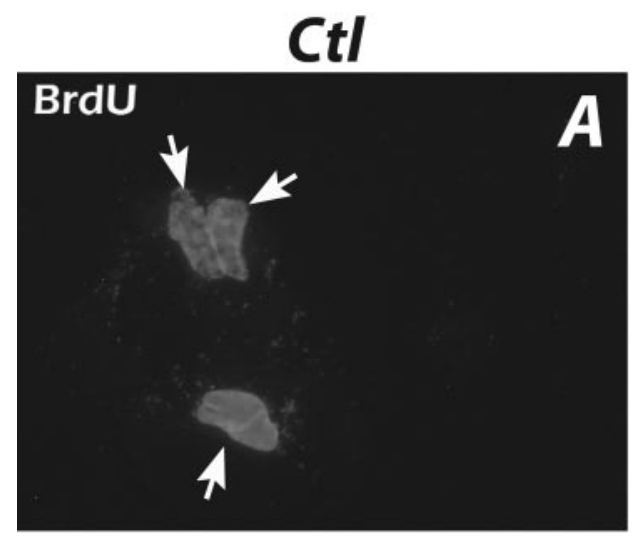

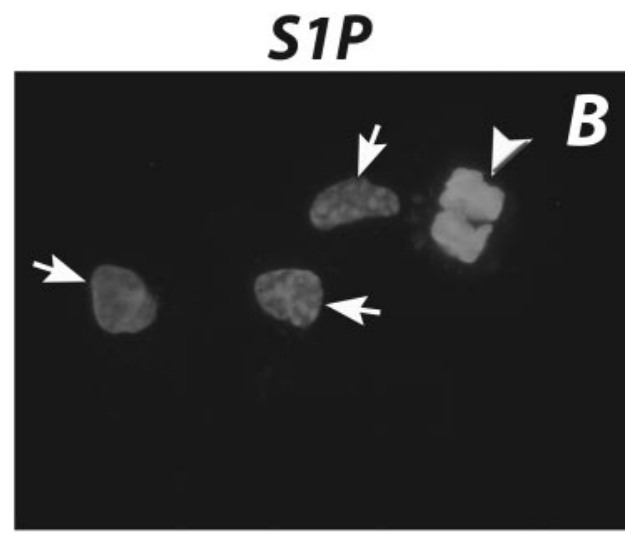

$\boldsymbol{E}$

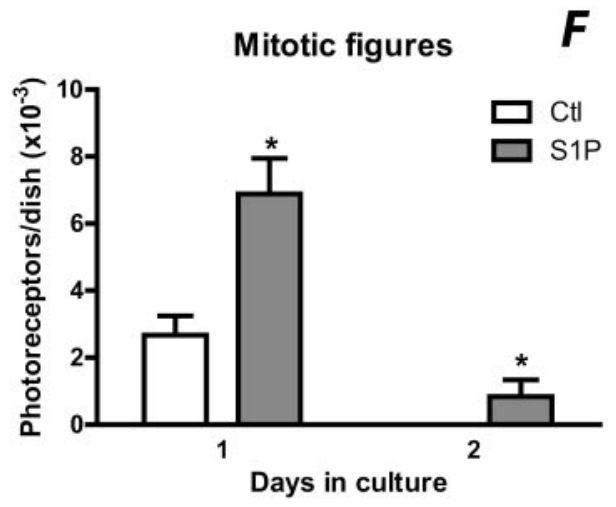

showed $11 \% \pm 0.3 \%$ opsin-positive photoreceptors (not shown) compared with $10.8 \% \pm 0.2 \%$ and $10.8 \% \pm 0.03 \%$, in S1P and DHA-supplemented cultures (Fig. 2A), respectively.

The increase in the percentage of opsin-positive photoreceptors was visible in a small range of S1P concentrations (Fig. 2B); $0.5 \mu \mathrm{M}$ S1P did not increase opsin expression, whereas 1 and $2 \mu \mathrm{M} \mathrm{S1P}$ augmented it similarly, from nearly $4 \%$ to approximately $8 \%$ (Fig. 2B). At $2.5 \mu \mathrm{M}$, S1P concentration was already deleterious for the cells (not shown). Western blot analysis confirmed DHA, and S1P addition led to higher opsin levels compared with controls (Fig 2C).

We then explored the expression of peripherin, a structural protein found in disc rims in photoreceptor OS. S1P and DHA increased the percentage of peripherin-expressing photoreceptors approximately 30\% compared with controls (Fig. 2D). This increase in peripherin levels after the addition of S1P and DHA was evidenced clearly by Western blot analysis (Fig. 2E).

We then investigated the effect of S1P on the development of apical processes. As previously reported ${ }^{3,5}$ most photore- ceptors in control cultures lacked apical processes (Figs. 3A, 3D, 3G, 3J, 3M, 3P, 3R, 4), and opsin labeling was distributed throughout the whole cytoplasm and neurite (Figs. 3A, 3P, 3R). A small number of them had short cilia, showing acetylated $\alpha$-tubulin staining (Fig. 3G), and few were labeled with peripherin (Fig. 3D). S1P addition rapidly promoted the formation of apical processes and the localization in them of opsin (Fig. 3C) and peripherin (Figs. 3F, 3L, 3O). These peripherin-labeled processes (Figs. 3L, 3O, white arrows) were clearly observed protruding at the tip of cilia labeled with acetylated $\alpha$-tubulin (Figs. 3I, 3O, open arrows). Opsin localization in apical processes was evident in confocal micrographs (Figs. 3Q, 3S, white arrows). S1P effects on photoreceptor differentiation were similar to those of DHA (Figs. 3B, 3E, 3K, 3N). The higher percentage of photoreceptors with apical processes was already visible 4 hours after S1P or DHA addition and augmented continually after 2 days in vitro (Fig. 4A), doubling that in controls at every time point studied. The combined addition of S1P and DHA showed the same effect as each molecule by itself 
$\boldsymbol{A}$

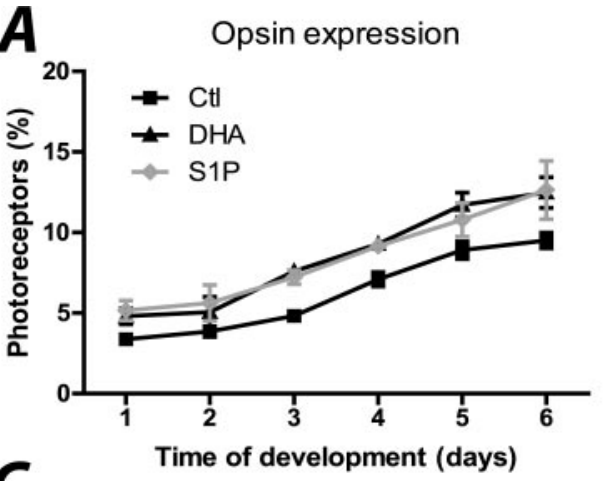

C

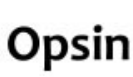

Actin

\section{(1)}
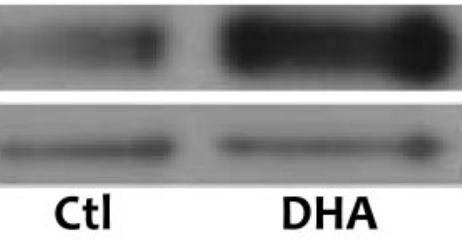

Peripherin Expression

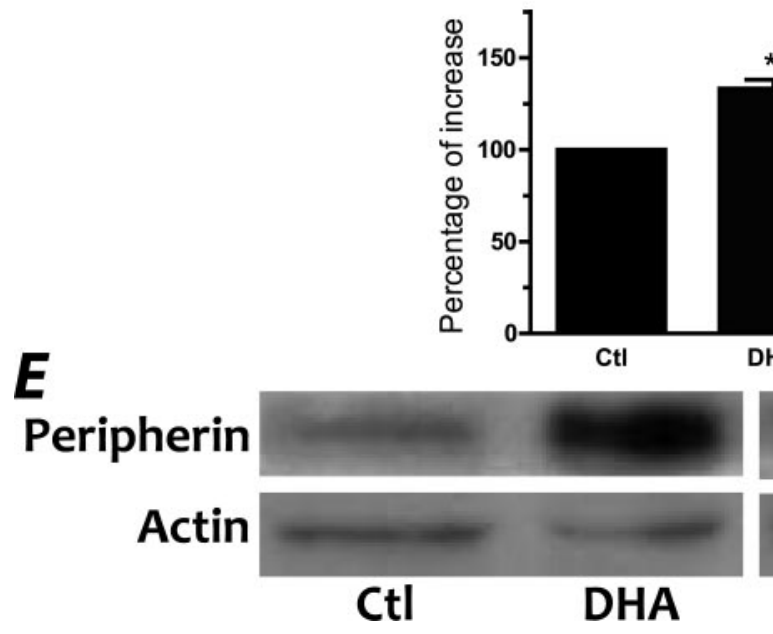

on the development of apical processes at day 6 , with $4.4 \% \pm$ $0.1 \%, 4.6 \% \pm 0.01 \%$, and $4.6 \% \pm 0.1 \%$ of photoreceptors having apical processes in S1P, DHA (Fig. 4A), and S1P plus DHA-supplemented cultures (not shown), respectively.

In controls, nearly $75 \%$ of peripherin-labeled photoreceptors had intensely labeled cilia, but few $(<29 \%)$ had peripherin labeled-apical processes (Fig. 4B). In contrast, in S1P- and DHA-supplemented cultures, most photoreceptors (approximately $70 \%$ ) showed peripherin-positive apical processes, and those with peripherin-labeled cilia decreased to 30\% (Fig. 4B). These results are evidence that $\mathrm{S} 1 \mathrm{P}$ and DHA further advanced photoreceptor differentiation.

\section{Brefeldin A Inhibition of DHA- and S1P-Induced Formation of Apical Processes}

To gain insight into the processes leading to OS formation, cultures were treated with BFA, which inhibits intracellular trafficking pathways, ${ }^{39}$ before DHA or S1P was added. Confocal micrographs confirmed that S1P and DHA promoted the development of opsin-labeled apical processes (white arrows in Figs. 5B, 5E, 5H, 5K), virtually absent in controls, in which opsin labeled photoreceptor neurites and cell bodies (thin
Opsin expression

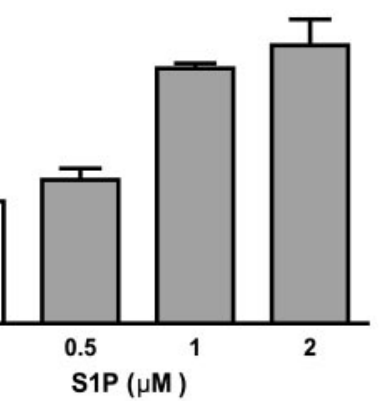
opsin and peripherin expression in photoreceptors. Neuronal cultures were supplemented at day 1 without (Ctl) or with $6.7 \mu \mathrm{M}$ DHA or S1P. The effect of time of development on the percentage of photoreceptors expressing opsin (A) was evaluated using $1 \mu \mathrm{M} \mathrm{S} 1 \mathrm{P}$; the effect of S1P concentration on opsin expression $(\mathbf{B})$ at day 6 in culture was determined at 0.5 to $2 \mu \mathrm{M}$ S1P. Opsin expression was evaluated by immunocytochemistry in cells fixed at day 1, 4 hours after the addition of S1P or DHA (indicated in Fig. as day 1), after 2 to 6 days of development, or by Western blot analysis (C) at day 6. The percentage of increase of photoreceptors expressing peripherin (D) in S1P-supplemented cultures compared with controls was evaluated at day 6 by immunocytochemistry, and peripherin levels were determined by Western blot analysis (E) with an anti-peripherin monoclonal antibody. Western blot analyses are representative of three independent experiments with similar results. ${ }^{*} P<$ 0.05 ; statistically significant differences compared with controls. arrows in Figs. 5A, 5D, 5G, 5J). BFA hindered DHA and S1P stimulatory effect, blocking this development and simultaneously increasing opsin delocalization (thin arrows in Figs. 5C, 5F, 5I, 5L). At day 4, the percentage of opsin-labeled photoreceptors with apical processes in DHA- and S1P-supplemented cultures was twice that in controls (Fig. 5M), and the addition of BFA reduced it almost to control levels (Fig. 5M), concurrently augmenting the percentage of photoreceptors having cilia (not shown). DHA and S1P increases in opsin expression were unaffected by BFA (Fig. $5 \mathrm{~N}$ ); the same percentage of opsin-expressing photoreceptors was found in DHA- and S1P-supplemented cultures with or without BFA. At these concentrations and lengths of incubation with BFA, no increase in photoreceptor apoptosis was observed (not shown).

\section{S1P Effect on Photoreceptor Apoptosis}

Photoreceptors cultured in media lacking their specific trophic factors developed normally for 3 to 4 days and then started to degenerate through an apoptotic pathway. ${ }^{5} \mathrm{~S} 1 \mathrm{P}$ addition significantly reduced photoreceptor apoptosis; after 6 days in vitro, approximately $50 \%$ of photoreceptors 
CtI
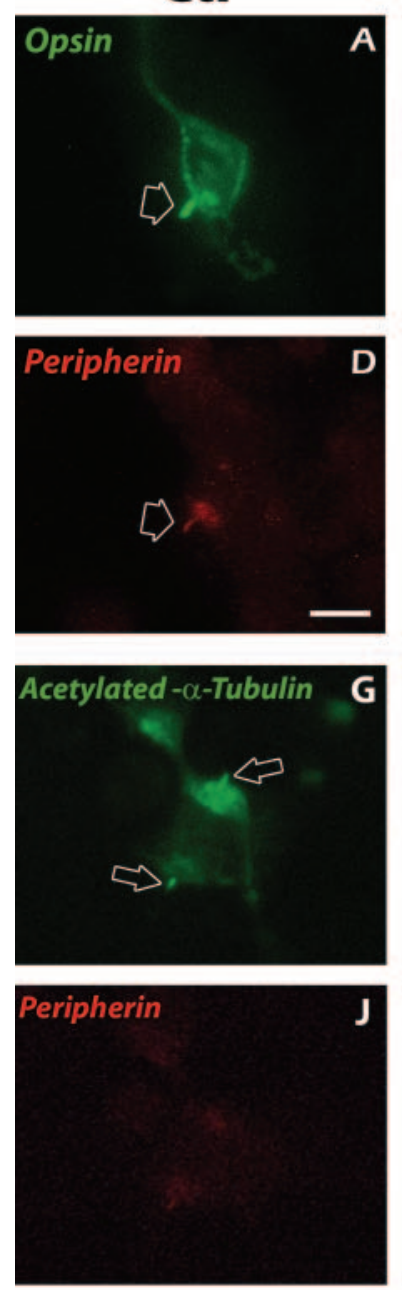

Figure 3. Effect of S1P on the formation of apical processes. Cultures were treated at day 1 with BSA (Ctl, left), DHA (middle), or S1P (right) and were fixed at day 6. Fluorescence micrographs show coimmunostaining of photoreceptors with opsin (A-C) and peripherin (D-F) antibodies and with acetylated $\alpha$-tubulin (G-I) and peripherin $(\mathbf{J}-\mathbf{L})$ antibodies and their merge (M-O). Intense staining with opsin and peripherin (thin arrows in $\mathbf{B}, \mathbf{C}, \mathbf{E}, \mathbf{F}$, $\mathbf{K}, \mathbf{L})$ was observed in apical processes in DHA and S1P-supplemented cultures but was present only in cilia in controls (wide arrows in $\mathbf{A}, \mathbf{D})$. Acetylated $\alpha$-tubulin-labeled photoreceptor cell bodies and cilia (open arrows in G-I, M-O) but did not label apical processes $(\mathbf{H}, \mathbf{I}$, $\mathbf{N}, \mathbf{O})$. Confocal micrographs (P-S) show opsin expression $(\mathbf{P}, \mathbf{Q})$ and the merge of this expression with Nomarsky images (R, S) in control $(\mathbf{P}, \mathbf{R})$ and S1P-supplemented $(\mathbf{Q}, \mathbf{S})$ cultures. Opsin was distributed throughout the cytoplasm and neurite in controls (open arrows in $\mathbf{P}$, $\mathbf{R})$, and S1P promoted its localization in photoreceptor apical processes (thin arrow in $\mathbf{Q}, \mathbf{S}$ ). Scale bars, 5 $\mu \mathrm{m}$.

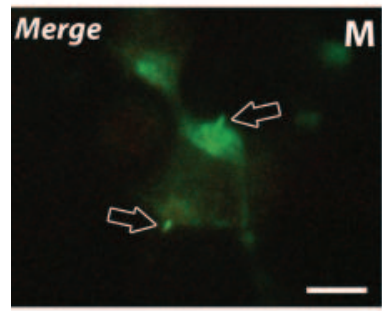

DHA

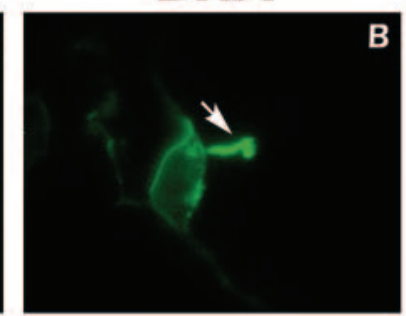

B
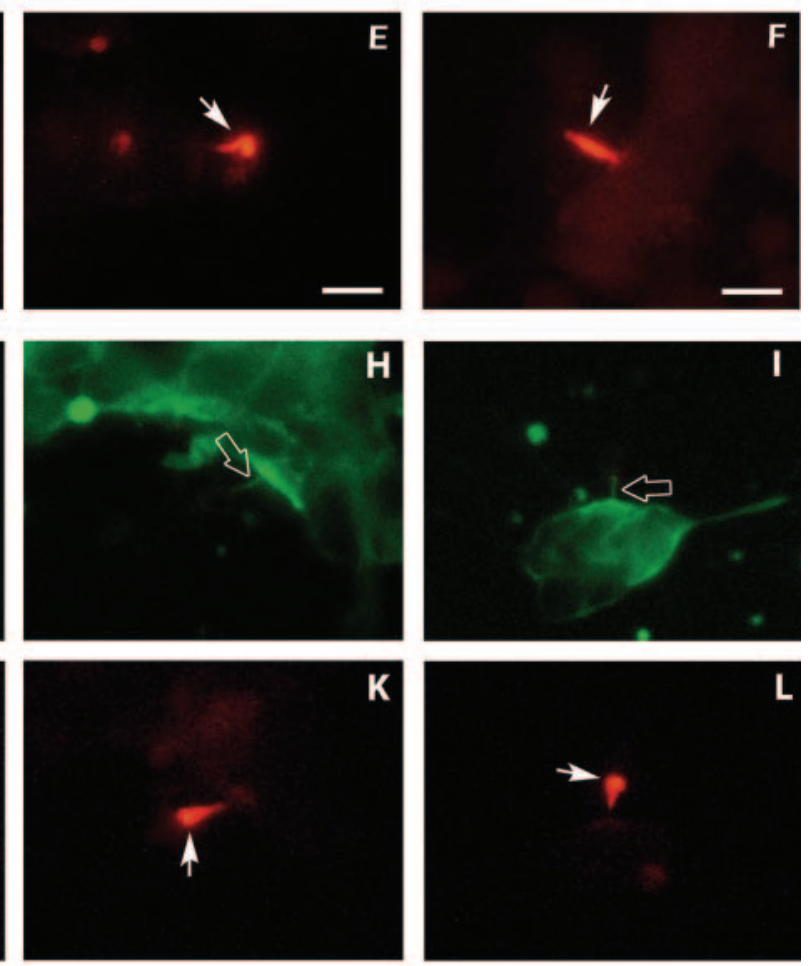

N
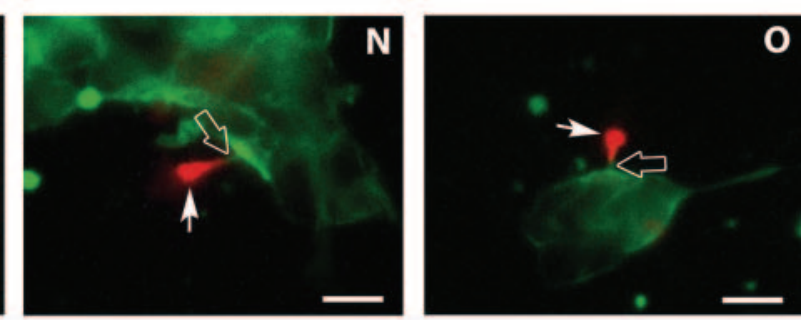

S1P
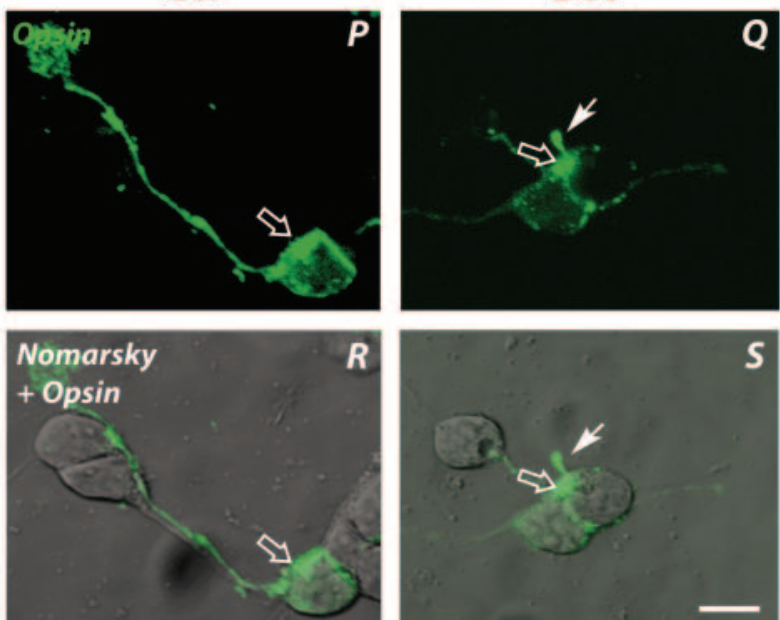
A

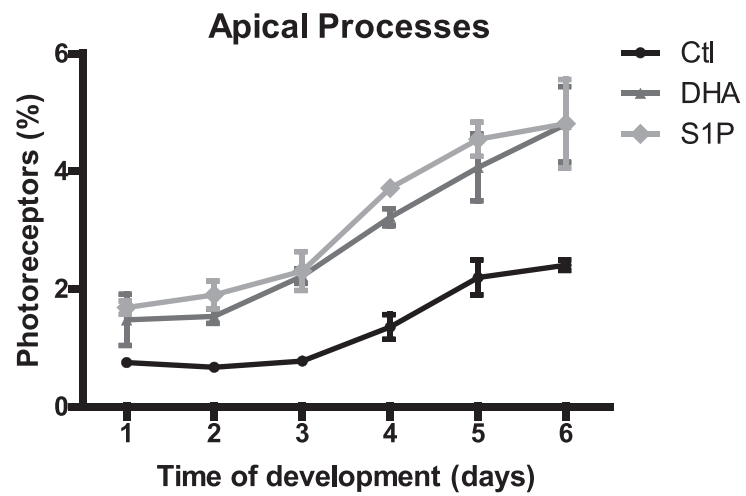

B

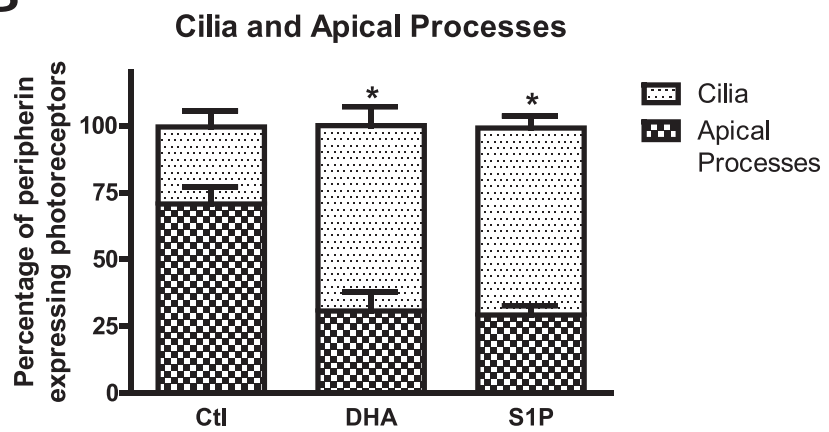

FIGURE 4. Effect of S1P and DHA on the development of apical processes. Neuronal cultures were supplemented at day 1 with BSA solution (Ctl), DHA, or S1P. Cells were fixed at day 1, 4 hours after the addition of S1P or DHA (indicated as day 1), after 2 to 6 days of development in vitro to evaluate the percentages of photoreceptors with apical processes (A), or at day 6 to evaluate the percentages of peripherin-expressing photoreceptors developing either only cilia or cilia plus apical processes (B). Note that in DHA- and S1P-treated cultures, more photoreceptors developed apical processes than only cilia, and the opposite was observed in control cultures. ${ }^{*} P<0.05$; statistically significant differences compared with controls.

were apoptotic in controls, and S1P decreased their apoptosis to less than $20 \%$ (Fig. 6). This decrease was confirmed by the reductions in the amounts of TUNEL-labeled photoreceptors in S1P-supplemented cultures compared with controls (not shown).

\section{Suppression of GDNF and DHA Effects by Inhibition of S1P Synthesis}

Previous work showed that GDNF stimulated the proliferation of photoreceptor progenitors. ${ }^{7}$ Our present data confirmed those results: approximately $26 \%$ of the cells took up BrdU in controls, and GDNF increased BrdU uptake to $40 \%$ of the cells (Fig. 7A). GDNF also increased the amount of mitotic figures from $3325 \pm 707$ to nearly $9013 \pm 1573$ per dish (Fig. 7B). To investigate whether S1P might be a mediator of the GDNF effect on photoreceptor proliferation, we inhibited S1P synthesis adding DHS, an SphK inhibitor, immediately after seeding the cells. Although the addition of DHS did not affect proliferation in day 1 control cultures (Figs. 7A, 7B), it completely inhibited GDNF-induced increases in proliferation (Figs. 7A, $7 \mathrm{~B})$, reducing BrdU uptake and the amount of mitotic figures to control values. These results strongly suggest that basal neuroblast proliferation was independent of S1P, but GDNF effect on proliferation required S1P synthesis.

We then investigated whether DHA required S1P synthesis to advance photoreceptor differentiation. In the absence of
DHS, DHA promoted the development of opsin-labeled apical processes (Figs. 8A, 8D), doubling the percentage of photoreceptors expressing opsin and having apical processes by day 6 (Figs. 8G, 8H). DHS addition had no effect on opsin expression and did not induce photoreceptor apoptosis in controls (not shown). DHS blocked the DHA effect on the formation of apical processes and the increase in opsin levels (Figs. 8B, 8E, $8 \mathrm{G}, 8 \mathrm{H}$ ) but did not affect those of S1P (Figs. 8G, 8H). S1P addition to cultures treated with DHS and DHA restored the increases in opsin expression and apical process development (Figs. 8C, 8F, 8G, 8H).

\section{DHA- and GDNF-Upregulated Sphingosine Kinase Expression}

Next, we investigated whether DHA and GDNF upregulated SphK levels in photoreceptors. Photoreceptors and amacrine neurons had a low and diffuse basal expression of SphK (Figs. 9A, 9F). GDNF addition to day 1 cultures increased the amount of photoreceptors expressing SphK 24 hours later, particularly in cultures treated with the proteosome inhibitor MG-132 (Figs. 9B, 9D). The higher SphK levels were confirmed by Western blot analysis (Fig. 9E).

To evaluate the effect of DHA on SphK expression in photoreceptors, 3-day cultures were treated with kainic acid to eliminate amacrine neurons. Almost no increase in SphK expression was visible after DHA addition in 4-day cultures because of its short lifetime (Fig. 9G). When cultures were treated with MG-132 to prevent SphK degradation, DHA enhancement of SphK expression was clearly evident (Fig. 9I). DHA upregulation of SphK levels was also evidenced by Western blot analysis (Fig. 9E).

In addition to the increased expression of SphK in DHA and GDNF-supplemented cultures, many photoreceptors localized this expression in their plasma membranes, which showed intense SphK labeling (thin arrows in Figs. 9D, 9I). This suggested that DHA and GDNF might promote the translocation of this enzyme from the cytosol to the plasma membrane, a mechanism activated by other trophic factors to induce SphK activation. ${ }^{40}$

\section{Discussion}

Uncovering cues for controlling the proliferation and development of retinal photoreceptors is essential for advancing the development of new tools for generating functional photoreceptors in neurodegenerative diseases of the retina. Our results demonstrate for the first time that S1P has a crucial role in regulating the proliferation and differentiation of photoreceptors. S1P stimulated the proliferation of photoreceptor progenitors at early culture times and advanced their differentiation as photoreceptors. These results also provide strong evidence that GDNF and DHA enhance the synthesis of S1P by enhancing the expression of SphK. Increased levels of S1P are required for GDNF and DHA stimulation of photoreceptor proliferation and differentiation, respectively, suggesting S1P might be an essential intracellular signal for controlling these processes.

\section{S1P as a Cue for the Proliferation of Photoreceptor Progenitors}

Most rod photoreceptors are born between postnatal day $(\mathrm{P}) 0$ and $\mathrm{P} 2$ in rodent retinas; they are the last neuronal type to be born in the retina in vivo. ${ }^{41,42}$ We have previously demonstrated that in neuronal cultures from day 0 rat retinas, photoreceptor progenitors accomplish their last rounds of mitosis in 
CtI
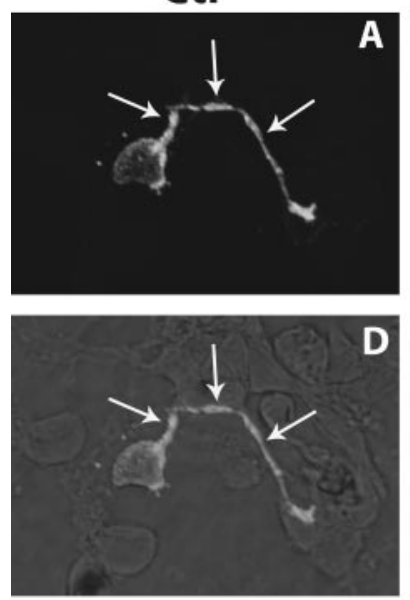

CtI
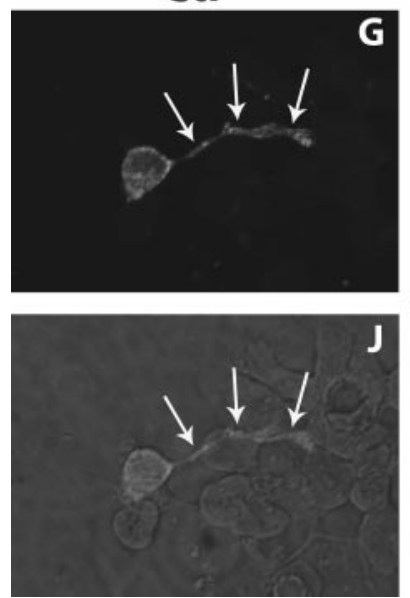

DHA
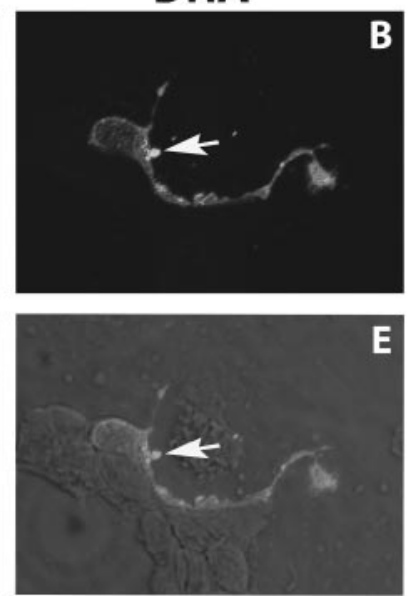

S1P
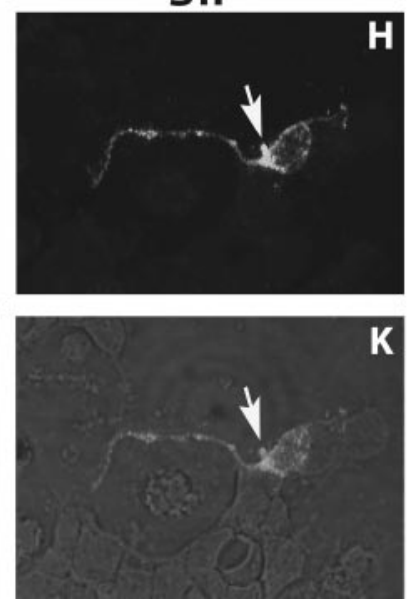

DHA + BFA
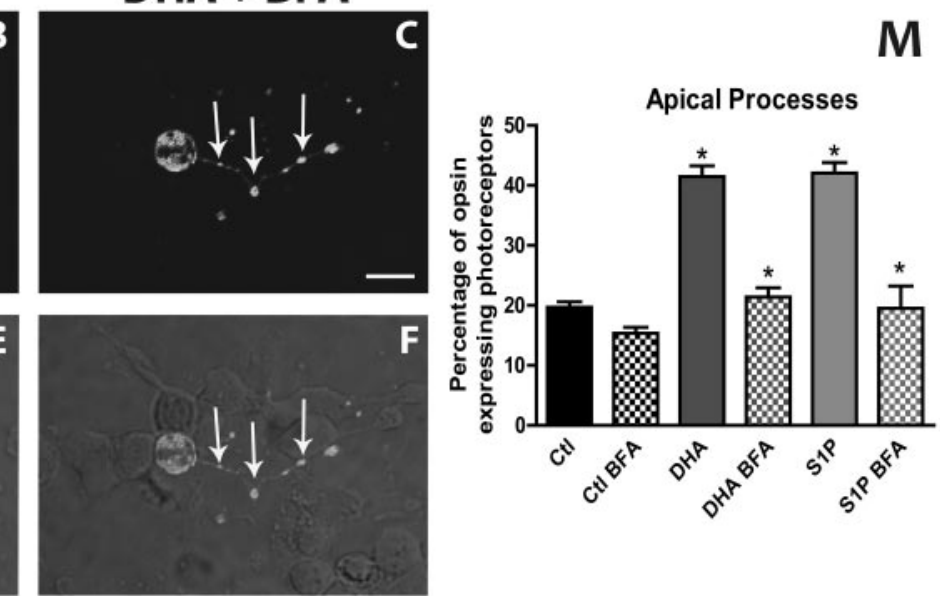

S1P + BFA
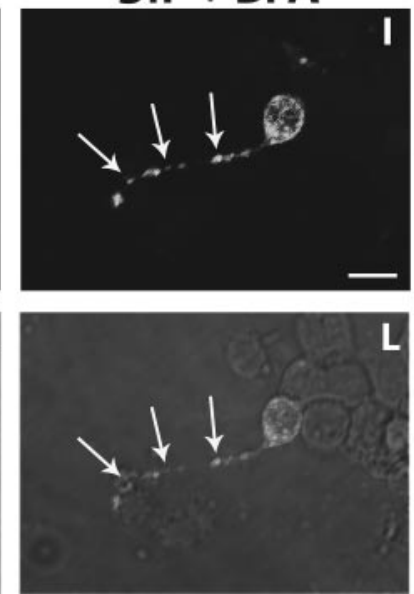

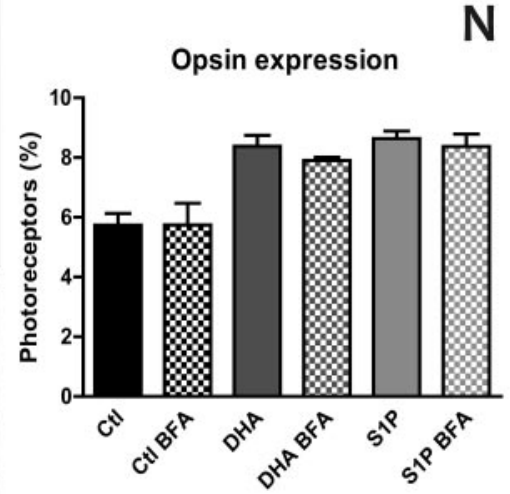

FIGURE 5. Brefeldin A inhibition of DHA and S1P effect on apical process formation. Day 2 neuronal cultures were treated with $0.5 \mu \mathrm{g} / \mathrm{mL}$ Brefeldin A and 2 hours later with either DHA or S1P; cultures were fixed at day 4. Fluorescence (A-C) and merge of fluorescence and phase confocal micrographs show opsin expression in control (A, D, G, J), DHA-supplemented (B, E), or S1P-supplemented (H, K) cultures and cultures treated with BFA and then with either DHA $(\mathbf{C}, \mathbf{F})$ or S1P $(\mathbf{I}, \mathbf{L})$. DHA and S1P promoted the development of opsin-labeled apical processes (white arrow in $\mathbf{B}, \mathbf{E}, \mathbf{H}, \mathbf{K})$, and the addition of BFA prevented their development in spite of the addition of DHA and S1P (C, F, I, L). Note that in control and BFA-treated cultures, opsin was widely distributed over the whole cell membrane and neurites (thin arrows in $\mathbf{A}, \mathbf{D}, \mathbf{C}, \mathbf{F}, \mathbf{G}, \mathbf{J}, \mathbf{I}, \mathbf{L}$ ). Scale bars, $5 \mu \mathrm{m}$. The percentage of opsin-labeled photoreceptors showing apical processes $(\mathbf{M})$ and expressing opsin (N) were determined by immunocytochemistry. ${ }^{*} P<0.05$; statistically significant differences compared with controls.

vitro. DHA and GDNF have opposing roles in controlling this proliferation. DHA induces the exit of photoreceptor progenitors from the cell cycle, while GDNF has a mitogenic effect, promoting proliferation. ${ }^{7}$ Identifying the molecular cues that induce undifferentiated cells to proliferate and eventually differentiate as photoreceptors has become even more relevant since the recent finding of stem cells in the retina because they can provide a therapeutic alternative to replace lost neurons. $\mathrm{S} 1 \mathrm{P}$ is involved in the regulation of mammalian cell proliferation and growth in many tissues. ${ }^{12}$ In the nervous system, S1P induces the proliferation of embryonic neural progenitor cells, ${ }^{22}$ whereas SphK1/2 null mice show decreased mitogenesis and increased neuronal apoptosis in almost all brain regions. ${ }^{43}$ We show here for the first time that S1P stimulated the proliferation of photoreceptor progenitors at early times of development, prolonging their permanence in the cell cycle. Interestingly, S1P effects on proliferation were similar to those of $\mathrm{GDNF}^{7}$ suggesting that S1P might be a key signal in controlling photoreceptor proliferation.

\section{S1P in the Advancement of Photoreceptor Differentiation}

Once photoreceptor progenitors exit the cell cycle, their differentiation proceeds in vivo through several steps. They initially develop a distal connecting cilium and then start expressing opsin and accumulating membranes at the cilium tip, which will finally form the rhodopsin-containing disks and the OS characteristic of mature photoreceptors. This differentiation appeared to be arrested in vitro. Cultures lacking S1P and DHA had poor opsin expression, as diffusely distributed over the entire plasmalemma as that found in immature photoreceptors, which show opsin labeling in inner and outer segment membranes and in synaptic terminal. It has been proposed that this distribution results from an inefficient, immature machinery for opsin transport. ${ }^{44}$ Photoreceptors in control cultures failed to develop apical processes; some of them concentrated peripherin, the disc rim protein essential for the morphogenesis and maintenance of the OS, in their cilia. This also mimics 


\section{Apoptosis}

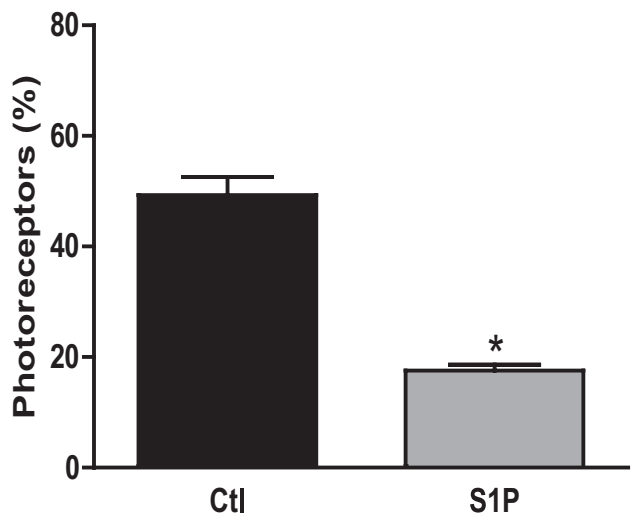

FIGURE 6. S1P prevention of photoreceptor apoptosis. Cultures were supplemented without (black bar) or with (gray bar) S1P at day 1 and were fixed at day 6 . The percentage of apoptotic photoreceptors was determined by quantifying the number of pyknotic or fragmented nuclei with DAPI. ${ }^{*} P<0.05$; statistically significant difference compared with control.

early development in vivo, during which peripherin is delivered to the site of OS morphogenesis before this morphogenesis is initiated. ${ }^{45}$ As a whole, this suggests that essential cues for advancing differentiation were absent in vitro.

We have previously demonstrated that DHA advances photoreceptor differentiation in vitro. ${ }^{3,5,38}$ We have now uncovered a key role for $\mathrm{S} 1 \mathrm{P}$ in enhancing this differentiation. The addition of S1P, and also of DHA, rapidly increased the expression of opsin and peripherin, induced the accumulation of membranes that resembled rudimentary OS or "apical processes" at the end of photoreceptor cilia, and promoted the colocalization of opsin and peripherin in the newly formed apical processes, as occurs during differentiation in vivo. Opsin and peripherin are essential for the development of normal OS. Underscoring the relevance of their increased expression in the development of OS, rhodopsin null mice and homozygous peripherin $/ r d s$ knockout mice that do not synthesize peripherin $/ r d s$ fail to form OS and undergo slow degeneration. ${ }^{46-48}$ Rhodopsin constitutes approximately $85 \%$ of the proteins forming photoreceptor OS, and injured rods lose the ability to localize opsin in the OS. ${ }^{49,50}$ Accumulating high concentrations of these proteins might be an essential prerequisite for building these specialized membrane structures. S1P and DHA advanced photoreceptor differentiation by increasing the synthesis of proteins characteristic of OS and by promoting the development of apical processes, initial steps in the formation of OS. DHA and S1P seemed to act on the same population of photoreceptors or to activate the same signaling pathways because the combined addition of both lipid molecules did not potentiate their effects.

To improve our understanding of these mechanisms, we inhibited intracellular trafficking with BFA. BFA did not affect the increase in opsin expression induced by S1P and DHA, but it almost completely blocked the formation of apical processes and opsin localization in them, keeping photoreceptors at the same differentiation stage as in controls. Opsin is synthesized in the endoplasmic reticulum, then modified in the Golgi, and is then vectorially transported in intracellular vesicles to the base of the connecting cilia for OS assemblage..$^{51-53}$ In frog photoreceptors, BFA does not affect opsin synthesis but reversibly inhibits its transport ${ }^{53}$ and that of DHA-containing phospholipids. ${ }^{54}$ Our results are consistent with a dual action of S1P and DHA. These molecules stimulate the synthesis of proteins essential for OS in a BFA-independent process. They also promote the morphogenesis of OS, targeting these proteins to them, a process that requires the intracellular trafficking of opsin-containing vesicles to the tip of photoreceptor cilia and that is inhibited by BFA. DHA represents approximately $50 \%$ of the acyl chains esterified in rod OS phospholipids. ${ }^{55}$ The increase in DHA content that accompanies its effects on photoreceptor differentiation in vitro suggested the requirement for DHA-containing phospholipids for the formation of apical processes. ${ }^{3}$ These phospholipids are closely associated with rhodopsin in the newly formed disc membranes ${ }^{56}$ and have been proposed to have a relevant role in rhodopsin trafficking. ${ }^{54} \mathrm{~S} 1 \mathrm{P}$ has also been proposed to participate in lipid and rhodopsin trafficking to OS in the frog retina, stimulating the uptake and esterification of DHA into phospholipids, followed by their increased delivery, together with rhodopsin, to the OS. ${ }^{23}$ Our results show that blocking S1P synthesis completely inhibited DHA-induced enhancement of opsin synthesis and apical process morphogenesis, maintaining photoreceptor differentiation in control levels. This suggests that S1P synthesis is essential for DHA effects and points to an indispensable role for S1P in the assembly of the OS.

A

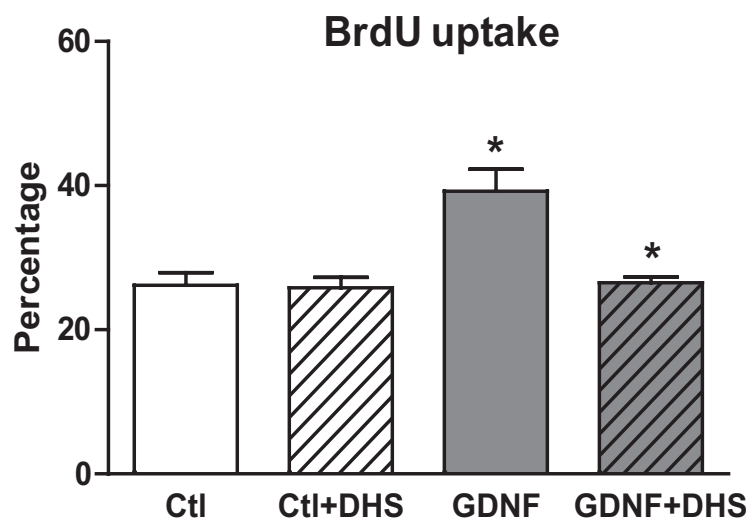

B

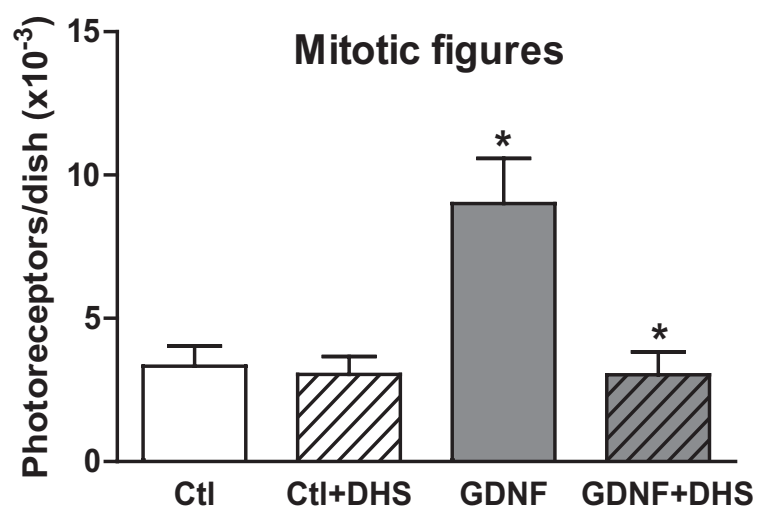

FIGURE 7. Effect of inhibition of S1P synthesis on GDNF-stimulated proliferation of photoreceptor progenitors. Neuronal cultures were treated without $(\mathrm{Ctl})$ or with $1 \mu \mathrm{M}$ DHS 1 hour after the cells were seeded and were supplemented without or with $4 \mathrm{nM}$ GDNF 1 hour later. BrdU was added to the cultures 4 hours later, and its uptake was determined after 18 hours. The percentage of BrdU-positive cells (A) and the amount of mitotic figures observed in photoreceptor progenitors (B) were determined by immunocytochemistry. ${ }^{*} P<0.05$; statistically significant differences compared with controls. 
DHA
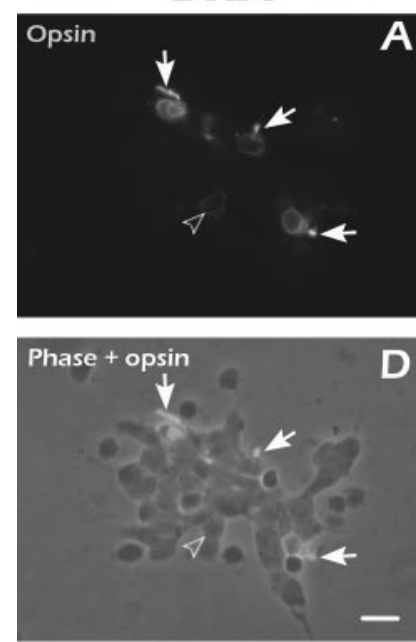

G

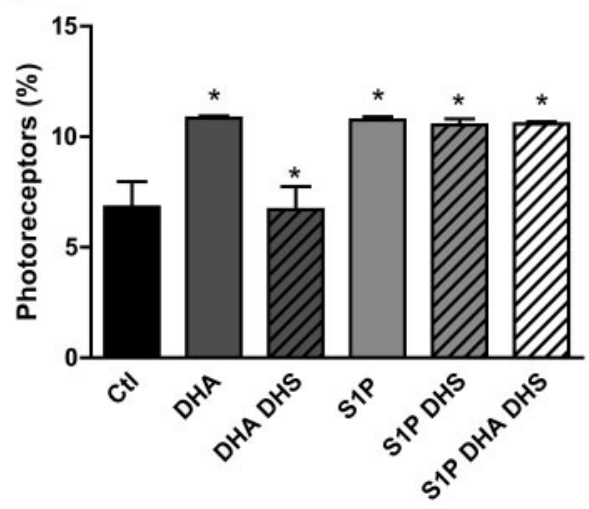

DHA + DHS + SIP
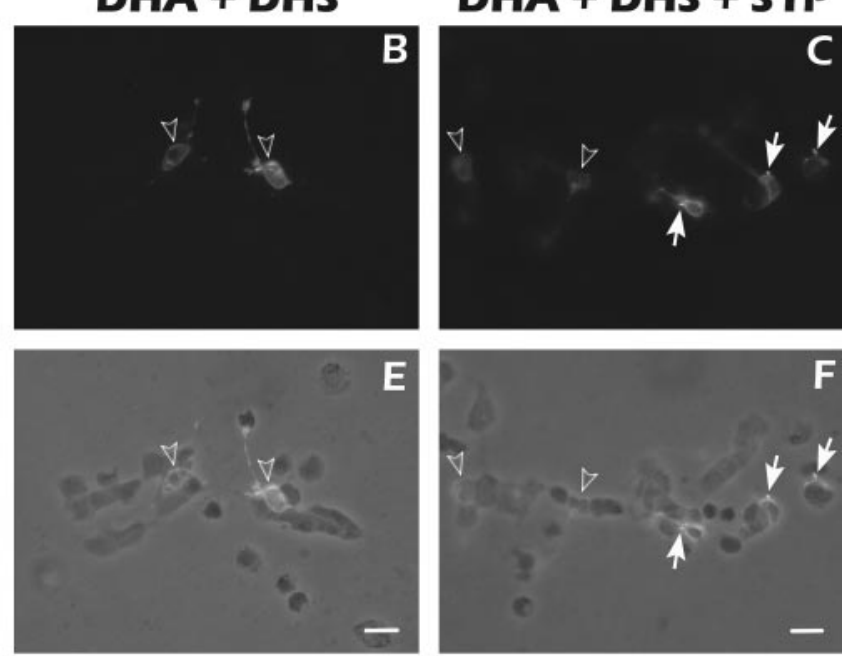

$\mathrm{H}$

Apical Processes

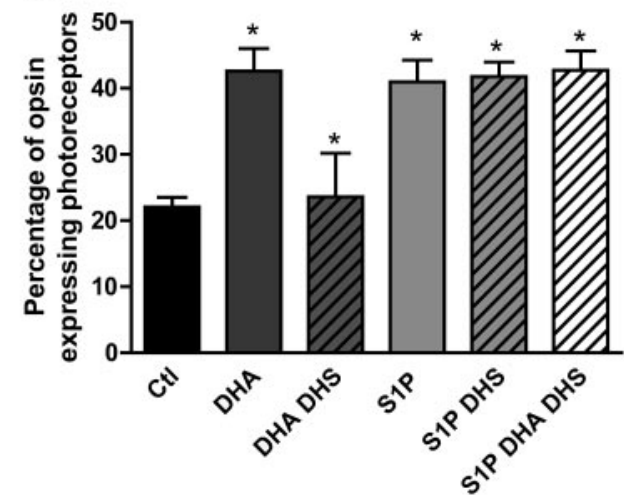

\section{S1P as a Promoter of Photoreceptor Survival}

Photoreceptors in our culture conditions start degenerating after 4 days in vitro, and trophic factors, such as DHA, prevent this degeneration. ${ }^{4,5,57} \mathrm{~S} 1 \mathrm{P}$ remarkably enhanced photoreceptor survival, thereby preventing apoptosis. S1P has been shown to suppress apoptosis in many cell types. ${ }^{20,58,59}$ Although few data exist on the nervous system, S1P has been reported to protect mesencephalic neurons against excitotoxicity. ${ }^{60} \mathrm{~S} 1 \mathrm{P}$ antiapoptotic effects have been associated with activation of the ERK/MAPK pathway, ${ }^{59}$ the same pathway activated by DHA to prevent photoreceptor death. ${ }^{14}$ Further work is required to establish which intracellular pathways are involved in $\mathrm{S} 1 \mathrm{P}$ protection. However, our results demonstrate that $\mathrm{S} 1 \mathrm{P}$ is involved in photoreceptor survival and suggest that DHA and S1P have similar effects not only on photoreceptor differentiation but on their survival as well.

\section{S1P as a Second Messenger for DHA and GDNF Signaling}

S1P is unique as a signaling molecule because it has two distinct mechanisms to mediate its biological effects. It acts as an extracellular ligand, binding to and activating G-proteinrelated membrane receptors, to regulate processes such as cytoskeletal rearrangements, cell migration, angiogenesis, and embryonic development of the heart. ${ }^{11}$ In mammals, S1P acts also as an intracellular second messenger in a receptor-independent manner. ${ }^{61}$ Activation of SphK leads to the accumulation of $\mathrm{S} 1 \mathrm{P}$, which then activates diverse downstream effectors, such as signaling pathways regulating calcium mobilization, DNA synthesis, cell growth, tumorigenesis, and suppression of apoptosis. ${ }^{19,61,62}$ Increasing evidence shows that there is mutual crosstalk between S1P and growth factor-activated signaling cascades. The overlap in the biological functions of S1P and those of DHA or GDNF prompted us to investigate whether these trophic factors might require the synthesis of S1P to exert their effects on photoreceptor development. Nothing is known concerning the regulation of S1P synthesis in the retina. Our results show that blocking S1P synthesis by inhibiting SphK1 activity completely blocked the GDNF mitogenic effect. Similarly, inhibiting SphK1 abolished DHA enhancement of opsin expression and apical process formation. The addition of S1P to DHA and DHS-supplemented cultures restored the increase in opsin levels and the development of apical processes. This strongly supports the proposal that GDNF and DHA might promote S1P synthesis and then use $\mathrm{S} 1 \mathrm{P}$ as a second messenger to mediate their biological effects.

SphK1 is a highly regulated enzyme; growth factors can transactivate S1P signaling, increasing S1P levels through activation or enhanced transcription of SphK, particularly the SphK1 isoform, or through the rapid activation and translocation of SphK1 from the cytosol to the plasma membrane. ${ }^{40,63}$ Cytokines and growth factors, such as transforming growth factor $\beta(\mathrm{TGF} \beta)$ and nerve growth factor (NGF), increase SphK1 expression in fibroblasts and PC12 cells, respectively. ${ }^{40,64}$ Our results demonstrate that GDNF and DHA increased SphK1 levels in photoreceptors, indicating that an upregulation of SphK1 transduction is among the mechanisms 

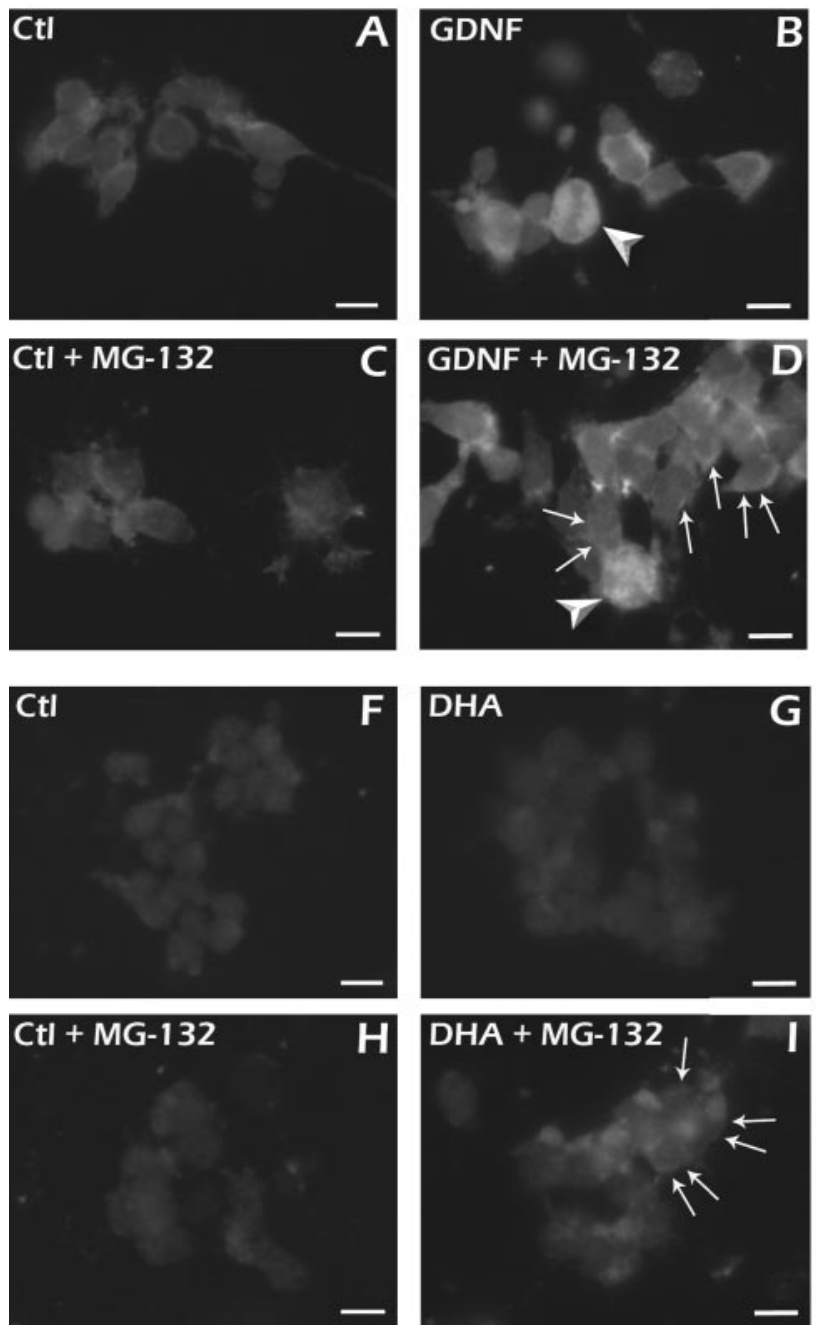

activated by DHA and GDNF to augment S1P synthesis. They also suggest that these factors might induce the translocation, and consequent activation, of SphK to the plasma membrane, implying that a combination of enhanced transcription and higher activity might contribute to augment S1P levels. SphK1 synthesizes S1P through phosphorylation of the sphingosine produced from ceramide hydrolysis. Given that S1P and its precursors, ceramide and sphingosine, have opposing roles in the control of cell survival and growth, with S1P implicated in cell survival and growth and ceramide involved in proapoptotic and growth-inhibitory effects, favoring the synthesis of either sphingolipid might have crucial effects on cell fate. Hence, SphK might have a key role in defining this balance; the upregulation of its expression by trophic factors such as GDNF and DHA would increase S1P levels, which would promote the proliferation of photoreceptor progenitors at early developmental times and then advance their differentiation, ensuring the morphogenesis of OS and the development of mature photoreceptors.

In conclusion, we propose that $\mathrm{S} 1 \mathrm{P}$ is a key mediator for regulating the final amount of photoreceptors in the retina, by initially controlling neuroblast proliferation and later promoting photoreceptor survival and differentiation. Photoreceptor trophic factors, such as GDNF and DHA, might elicit their biological effects by enhancing the synthesis of S1P, which would then act as a crucial second messenger for photoreceptor development.
E

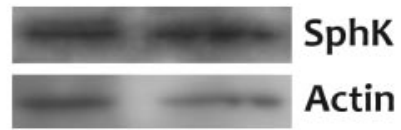

\section{CtI MG GDNF MG}

SK/Actin ratio

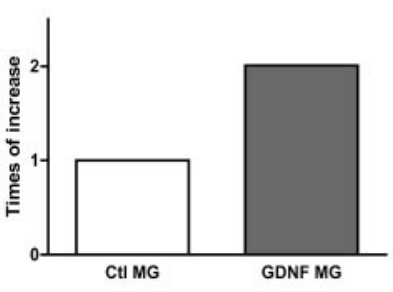

FIGURE 9. Effect of GDNF and DHA on sphingosine kinase (SphK) expression. Neuronal cultures from day 0 rat retinas were supplemented with GDNF, treated 5 hours later with 0.1 $\mu$ M MG-132, and fixed or lysed after 24 hours. Cultures from day 1 retinas were treated with kainic acid at day 3 to eliminate amacrine neurons, supplemented with DHA at day 4 with or without MG-

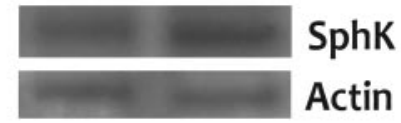

\section{CtI MG DHA MG}

SK/Actin ratio

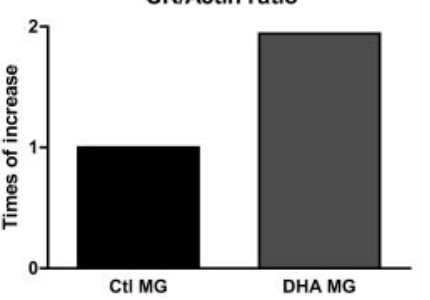

132, and fixed or lysed at day 5. Fluorescence micrographs (A-D, F-I) show SphK expression determined by immunocytochemistry in cultures without $(\mathbf{A}, \mathbf{C}, \mathbf{F}, \mathbf{H})$ and with GDNF $(\mathbf{B}, \mathbf{D})$ or DHA $(\mathbf{G}, \mathbf{I})$ and by Western blot analysis in lysates obtained from day 1 (E) and day 5 (J) cultures using a specific anti-SphK1 antibody. Note that many cells in DHA- and GDNFsupplemented cultures show a marked localization of SphK labeling in the plasma membrane (tbin arrows in $\mathbf{D}, \mathbf{I})$. Mitotic figures were clearly visible in GDNF-treated cultures (arrowbeads in B, D). Scale bars, $5 \mu \mathrm{m}$.

\section{Acknowledgment}

The authors thank Beatriz de los Santos for her excellent technical assistance.

\section{References}

1. LaVail MM, Yasumura D, Matthes MT, et al. Protection of mouse photoreceptors by survival factors in retinal degenerations. Invest Ophthalmol Vis Sci. 1998;39:592-602.

2. Frasson M, Picaud S, Leveillard T, et al. Glial cell line-derived neurotrophic factor induces histologic and functional protection of rod photoreceptors in the $\mathrm{rd} / \mathrm{rd}$ mouse. Invest Ophthalmol Vis Sci. 1999; 40:2724-2734.

3. Rotstein NP, Politi LE, Aveldano MI. Docosahexaenoic acid promotes differentiation of developing photoreceptors in culture. Invest Ophthalmol Vis Sci. 1998;39:2750-2758.

4. Rotstein NP, Aveldano MI, Barrantes FJ, Roccamo AM, Politi LE. Apoptosis of retinal photoreceptors during development in vitro: protective effect of docosahexaenoic acid. J Neurochem. 1997;69: 504-513.

5. Rotstein NP, Aveldano MI, Barrantes FJ, Politi LE. Docosahexaenoic acid is required for the survival of rat retinal photoreceptors in vitro. J Neurochem. 1996;66:1851-1859.

6. Politi LE, Rotstein NP, Carri NG. Effect of GDNF on neuroblast proliferation and photoreceptor survival: additive protection with docosahexaenoic acid. Invest Ophthalmol Vis Sci. 2001;42:30083015 . 
7. Insua MF, Garelli A, Rotstein NP, German OL, Arias A, Politi LE. Cell cycle regulation in retinal progenitors by glia-derived neurotrophic factor and docosahexaenoic acid. Invest Opbthalmol Vis Sci. 2003; 44:2235-2244.

8. Maceyka M, Milstien S, Spiegel S. Sphingosine kinases, sphingosine-1-phosphate and sphingolipidomics. Prostaglandins Other Lipid Mediat. 2005;77:15-22.

9. Saba JD. Lysophospholipids in development: miles apart and edging in. J Cell Biochem. 2004;92:967-992.

10. Taha TA, Mullen TD, Obeid LM. A house divided: ceramide, sphingosine, and sphingosine-1-phosphate in programmed cell death. Biochim Biopbys Acta. 2006;1758:2027-2036.

11. Lahiri S, Futerman AH. The metabolism and function of sphingolipids and glycosphingolipids. Cell Mol Life Sci. 2007;64:22702284.

12. Kono M, Allende ML, Proia RL. Sphingosine-1-phosphate regulation of mammalian development. Biochim Biophys Acta. 2008; 1781:435-441.

13. Sabourdy F, Kedjouar B, Sorli SC, et al. Functions of sphingolipid metabolism in mammals-lessons from genetic defects. Biochim Biophys Acta. 2008;1781:145-183.

14. German OL, Miranda GE, Abrahan CE, Rotstein NP. Ceramide is a mediator of apoptosis in retina photoreceptors. Invest Ophthalmol Vis Sci. 2006;47:1658-1668.

15. Sanvicens N, Cotter TG. Ceramide is the key mediator of oxidative stress-induced apoptosis in retinal photoreceptor cells. $J$ Neurochem. 2006;98:1432-1444.

16. Spiegel S, Milstien S. Sphingosine 1-phosphate, a key cell signaling molecule. J Biol Chem. 2002;277:25851-25854.

17. Saba JD, Hla T. Point-counterpoint of sphingosine 1-phosphate metabolism. Circ Res. 2004;94:724-734.

18. Taha TA, Kitatani K, El-Alwani M, Bielawski J, Hannun YA, Obeid LM. Loss of sphingosine kinase- 1 activates the intrinsic pathway of programmed cell death: modulation of sphingolipid levels and the induction of apoptosis. FASEB J. 2006;20:482- 484.

19. Van B Jr, Lee MJ, Menzeleev R, et al. Dual actions of sphingosine1-phosphate: extracellular through the Gi-coupled receptor Edg-1 and intracellular to regulate proliferation and survival. J Cell Biol. 1998;142:229-240.

20. Lebman DA, Spiegel S. Cross-talk at the crossroads of sphingosine1-phosphate, growth factors, and cytokine signaling. J Lipid Res. 2008; 49:1388-1394.

21. McGiffert C, Contos JJ, Friedman B, Chun J. Embryonic brain expression analysis of lysophospholipid receptor genes suggests roles for $\operatorname{sip}(1)$ in neurogenesis and $\operatorname{sip}(1-3)$ in angiogenesis. FEBS Lett. 2002;531:103-108.

22. Harada J, Foley M, Moskowitz MA, Waeber C. Sphingosine-1-phosphate induces proliferation and morphological changes of neural progenitor cells. J Neurochem. 2004;88:1026-1039.

23. Deretic D, Traverso V, Parkins N, Jackson F, Rodriguez de Turco EB, Ransom N. Phosphoinositides, ezrin/moesin, and rac1 regulate fusion of rhodopsin transport carriers in retinal photoreceptors. Mol Biol Cell. 2004;15:359-370.

24. Pasquare SJ, Salvador GA, Giusto NM. Involvement of lysophosphatidic acid, sphingosine 1-phosphate and ceramide 1-phosphate in the metabolization of phosphatidic acid by lipid phosphate phosphatases in bovine rod outer segments. Neurochem Res. 2008;33:1205-1215.

25. Bobrow MN, Shaughnessy KJ, Litt GJ. Catalyzed reporter deposition, a novel method of signal amplification, II: application to membrane immunoassays. J Immunol Metbods. 1991;137:103112.

26. Hopman AH, Ramaekers FC, Speel EJ. Rapid synthesis of biotin-, digoxigenin-, trinitrophenyl-, and fluorochrome-labeled tyramides and their application for In situ hybridization using CARD amplification. J Histochem Cytochem. 1998;46:771-777.

27. Politi LE, Bouzat C, de los Santos EB, Barrantes FJ. Heterologous retinal cultured neurons and cell adhesion molecules induce clustering of acetylcholine receptors and polynucleation in mouse muscle BC3H-1 clonal cell line. J Neurosci Res. 1996;43:639-651.
28. Adler R. Regulation of neurite growth in purified retina neuronal cultures: effects of PNPF, a substratum-bound, neurite-promoting factor. J Neurosci Res. 1982;8:165-177.

29. Politi L, Rotstein N, Carri N. Effects of docosahexaenoic acid on retinal development: cellular and molecular aspects. Lipids. 2001; 36:927-935.

30. Barnstable CJ. Monoclonal antibodies which recognize different cell types in the rat retina. Nature. 1980;286:231-235.

31. Molday RS, Hicks D, Molday L. Peripherin. A rim-specific membrane protein of rod outer segment discs. Invest Ophthalmol Vis Sci. $1987 ; 28: 50-61$.

32. Uchihara T, Nakamura A, Nagaoka U, Yamazaki M, Mori O. Dual enhancement of double immunofluorescent signals by CARD: participation of ubiquitin during formation of neurofibrillary tangles. Histochem Cell Biol. 2000;114:447-451.

33. Abrams L, Politi LE, Adler R. Differential susceptibility of isolated mouse retinal neurons and photoreceptors to kainic acid toxicity: in vitro studies. Invest Ophthalmol Vis Sci. 1989;30:2300-2308.

34. Buehrer BM, Bell RM. Inhibition of sphingosine kinase in vitro and in platelets: implications for signal transduction pathways. $\mathrm{J} \mathrm{Biol}$ Chem. 1992;267:3154-3159.

35. Liu H, Sugiura M, Nava VE, et al. Molecular cloning and functional characterization of a novel mammalian sphingosine kinase type 2 isoform. J Biol Chem. 2000;275:19513-19520.

36. Lee DH, Goldberg AL. Proteasome inhibitors: valuable new tools for cell biologists. Trends Cell Biol. 1998;8:397-403.

37. Laemmli UK, Beguin F, Gujer-Kellenberger G. A factor preventing the major head protein of bacteriophage $\mathrm{T} 4$ from random aggregation. J Mol Biol. 1970;47:69-85.

38. Garelli A, Rotstein NP, Politi LE. Docosahexaenoic acid promotes photoreceptor differentiation without altering Crx expression. Invest Ophthalmol Vis Sci. 2006;47:3017-3027.

39. Lippincott-Schwartz J, Donaldson JG, Schweizer A, et al. Microtubule-dependent retrograde transport of proteins into the ER in the presence of brefeldin A suggests an ER recycling pathway. Cell. 1990;60:821-836.

40. Toman RE, Payne SG, Watterson KR, et al. Differential transactivation of sphingosine-1-phosphate receptors modulates NGF-induced neurite extension. J Cell Biol. 2004;166:381-392.

41. Young RW. Cell proliferation during postnatal development of the retina in the mouse. Brain Res. 1985;353:229-239.

42. Cepko CL, Austin CP, Yang X, Alexiades M, Ezzeddine D. Cell fate determination in the vertebrate retina. Proc Natl Acad Sci USA. 1996;93:589-595.

43. Mizugishi K, Yamashita T, Olivera A, Miller GF, Spiegel S, Proia RL. Essential role for sphingosine kinases in neural and vascular development. Mol Cell Biol. 2005;25:11113-11121.

44. Nir I, Cohen D, Papermaster DS. Immunocytochemical localization of opsin in the cell membrane of developing rat retinal photoreceptors. J Cell Biol. 1984;98:1788-1795.

45. Lee ES, Burnside B, Flannery JG. Characterization of peripherin/rds and rom-1 transport in rod photoreceptors of transgenic and knockout animals. Invest Ophthalmol Vis Sci. 2006;47:21502160.

46. Sanyal S, De Ruiter A, Hawkins RK. Development and degeneration of retina in rds mutant mice: light microscopy. J Comp Neurol. 1980;194:193-207.

47. Lem J, Krasnoperova NV, Calvert PD, et al. Morphological, physiological, and biochemical changes in rhodopsin knockout mice. Proc Natl Acad Sci US A. 1999;96:736-741.

48. Humphries MM, Rancourt D, Farrar GJ, et al. Retinopathy induced in mice by targeted disruption of the rhodopsin gene. Nat Genet. 1997; 15:216-219.

49. Nir I, Sagie G, Papermaster DS. Opsin accumulation in photoreceptor inner segment plasma membranes of dystrophic RCS rats. Invest Ophthalmol Vis Sci. 1987;28:62-69.

50. Nir I, Agarwal N, Sagie G, Papermaster DS. Opsin distribution and synthesis in degenerating photoreceptors of rd mutant mice. Exp Eye Res. 1989;49:403-421. 
51. Besharse JC, Pfenninger KH. Membrane assembly in retinal photoreceptors, I: freeze-fracture analysis of cytoplasmic vesicles in relationship to disc assembly. J Cell Biol. 1980;87:451-463.

52. Papermaster DS, Schneider BG, Besharse JC. Vesicular transport of newly synthesized opsin from the Golgi apparatus toward the rod outer segment: ultrastructural immunocytochemical and autoradiographic evidence in Xenopus retinas. Invest Ophthalmol Vis Sci. 1985;26:1386-1404.

53. Deretic D, Papermaster DS. Polarized sorting of rhodopsin on post-Golgi membranes in frog retinal photoreceptor cells. $J$ Cell Biol. 1991;113:1281-1293.

54. Rodriguez de Turco EB, Deretic D, Bazan NG, Papermaster DS Post-Golgi vesicles cotransport docosahexaenoyl-phospholipids and rhodopsin during frog photoreceptor membrane biogenesis. J Biol Chem. 1997;272:10491-10497.

55. Fliesler SJ, Anderson RE. Chemistry and metabolism of lipids in the vertebrate retina. Prog Lipid Res. 1983;22:79-131.

56. Gordon WC, Bazan NG. Docosahexaenoic acid utilization during rod photoreceptor cell renewal. J Neurosci. 1990;10:2190-2202.

57. German OL, Insua MF, Gentili C, Rotstein NP, Politi LE. Docosahexaenoic acid prevents apoptosis of retina photoreceptors by activating the ERK/MAPK pathway. J Neurochem. 2006;98:15071520 .

58. Edsall LC, Cuvillier O, Twitty S, Spiegel S, Milstien S. Sphingosine kinase expression regulates apoptosis and caspase activation in PC12 cells. J Neurochem. 2001;76:1573-1584.

59. Cuvillier O, Pirianov G, Kleuser B, et al. Suppression of ceramidemediated programmed cell death by sphingosine-1-phosphate. $\mathrm{Na}$ ture. 1996;381:800-803.

60. Shinpo K, Kikuchi S, Moriwaka F, Tashiro K. Protective effects of the TNF-ceramide pathway against glutamate neurotoxicity on cultured mesencephalic neurons. Brain Res. 1999;819:170-173.

61. Spiegel S, Milstien S. Exogenous and intracellularly generated sphingosine 1-phosphate can regulate cellular processes by divergent pathways. Biochem Soc Trans. 2003;31:1216-1219.

62. Young KW, Nahorski SR. Sphingosine 1-phosphate: a Ca2+ release mediator in the balance. Cell Calcium. 2002;32:335-341.

63. Wattenberg BW, Pitson SM, Raben DM. The sphingosine and diacylglycerol kinase superfamily of signaling kinases: localization as a key to signaling function. J Lipid Res. 2006;47:1128-1139.

64. Yamanaka M, Shegogue D, Pei H, et al. Sphingosine kinase 1 (SPHK1) is induced by transforming growth factor-beta and mediates TIMP-1 up-regulation. J Biol Chem. 2004;279:53994-54001. 